\title{
Short and medium term glycaemic control after pancreaticoduodenectomy
}

\author{
R.C. Siriwardana', L.M.P.M. Bandara ${ }^{1}$, N. Atulugama ${ }^{2}$, M.B. Gunathilke ${ }^{1}$, C.S. Ekanayake \\ 1 Department of Surgery, Faculty of Medicine, University of Kelaniya, Sri Lanka. \\ 2 National Cancer Hospital, Maharagama Sri Lanka
}

Key words: Pancreatoduodenectomy; diabetes mellitus

\begin{abstract}
\section{Introduction}

Perioperative outcomes of pancreaticoduodenectomy (PD) have improved over the years. Glycaemic control in longterm survivors is a matter of concern.
\end{abstract}

\section{Materials and methods}

48 surviving patients of 66 patients who underwent Whipple surgery from 2011 to 2015 were evaluated. Patients with recurrences, who had chemotherapy within three months and patients who had not completed a minimum six months follow-up were excluded. 25 patients were selected. Patients' demographic data, HbA1c level, fasting blood sugar level, physical activity index and waist to hip ratio were calculated. Volume of the pancreatic specimen was calculated. Non diabetics underwent oral glucose tolerance test (OGTT).

\section{Results}

There were 6 (24\%) pre-existing diabetics, 3 new onset diabetics and two patients with impaired glucose tolerance (20\%). Median preoperative BMI, body fat distribution, calculated median pancreatic volumes resected or underlying pancreatic pathology did not differ in diabetic and non-diabetic groups. In non-diabetics, HbAlc level or two hour OGTT did not associate with age, preoperative BMI, waist to hip ratio and resected pancreatic volumes.

\section{Conclusion}

Significant proportion of patients develop diabetes immediately after PD. Reliable prediction of this group pre-operatively is difficult due to many interacting, confounding factors. They need close monitoring in immediate perioperative period.

Correspondence: R.C. Siriwardana

E-mail: rohansiriwardana@yahoo.com

DOI: http://10.4038/sljs.v34i4.8315

The Sri Lanka Journal of Surgery 2016; 34(4): 11-15

\section{Introduction}

Pancreaticoduodenectomy (PD) is an aggressive treatment option for pathologies confined to the periampullary and pancreatic head region. Operative mortality after such surgery has decreased dramatically, rendering more long term survivors than in the past [1]. However, factors such as postoperative quality of life and post-PD glycaemic control remains matters of concern in the long-term survivors.

Pancreas is a mixed gland with both endocrine and exocrine components. PD reduces the volume of its beta-cells, thus lowering the threshold for pancreatogenic diabetes [2]. In addition, underlying chronic pancreatitis (CP), hyperlipidaemia, hypertension, increased BMI, fat distribution in the body, nutrition and physical activity are other confounding factors well known to increase insulin resistance and these may have a synergistic effect on post-PD diabetes [3]. In contrast, a few studies have shown to improve insulin resistance after PD [4].

There is limited data on post-PD glycaemic control and fluctuation in patients who survive the initial phase of treatment. Hence this study was focused on the changes in post - PD glycaemic control in the short and medium term, and to evaluate factors contributing to increased insulin resistance postoperatively in a cohort of patients who completed a minimum of six months follow-up after surgery.

\section{Materials and methods}

A cross-sectional analysis of 48 surviving patients who underwent PD from March 2011 to March 2015, at the North Colombo Teaching Hospital Faculty of Medicine, Ragama, Sri Lanka was performed to assess their glycaemic status. The patients who had i) post-operative pancreas related complications, ii) chemotherapy within three months after $P D$, iii) patients with recurrence and iv) patients who has not completed a minimum of six months follow-up were excluded.

In total 25 patients (55 years (range 18 - 77), $12(48 \%)$ females and $13(52 \%)$ males) who fulfilled the criteria with a median follow up period of 17 months (range 6 -42) month at the cross-sectional analytical point 
were chosen. All patients included in the study consented to participate and the study was approved by the institutional Ethics Review Committee.

All patients underwent standard Whipple surgical procedure. Reconstruction was done using a single jejunal loop with initial end to side pancreatico-jejunostomy followed by hepatico-jejunostomy. Posterior gastro-jejunostomy was then created $70 \mathrm{~cm}$ from the hepatico - jejunostomy through the transverse mesocolon. After completion the anastomosis was taken to the infracolic compartment.

There were six patients who were known pre-PD diabetics and they remained as diabetics during the postoperative surveillance period. New onset diabetes was detected in five $(20 \%)$ patients in the immediate postoperative period. All diagnosed diabetics were referred to the endocrinologist for optimal glycaemic control.

All study participants demonstrated good study compliance throughout the evaluation period. At a median period of 17 months, all 25 patients were subjected to fasting blood sugar (FBS) and glycosylated haemoglobin levels (HbA1C) assessments to evaluate whether the glycaemic status had deteriorated, improved or remained static.

The patients who were not known to have diabetes underwent oral glucose tolerance test (OGTT) and impaired glucose tolerance (IGT) status was diagnosed according to World Health Organization diagnostic criteria.

In all patients' basic information, hypoglycemic medication requirement, body mass index $(\mathrm{BMI})$, waist to hip ratio $(\mathrm{W} / \mathrm{H})$ and physical activity index (PAI) were recorded. Postsurgical physical activity was assessed using the 'General practice physical activity questionnaire' published by department of health, England [5]. Histopathology details were also recorded and pancreatic volume resected was quantified using the dimensions (length, width and height) of the resected specimen.
New onset diabetes and non-diabetics were compared retrospectively on the possible predictors for the development of diabetes in the immediate postoperative period. Preoperative BMI, age, waist to hip ratios, pancreatic volumes resected and the physical activity index were considered as covariates.

Post-PD Non diabetic groups at the median time of 17 months follow up were also evaluated on preoperative $\mathrm{BMI}, \mathrm{W} / \mathrm{H}$ and resected pancreatic volumes to predict progression to poor glycaemic status.

Chi-square test and the Mann-Whitney U test were used to compare variables where appropriate. Spearman's rank-order correlation with SPSS 22.0 version. $\mathrm{P}$ value of $<0.05$ was considered as statistically significant.

\section{Results}

The study cohort comprised 12 (48\%) females and 13 (52\%) males with a median age of 55 years (range 18-77) and a BMI of $20.92 \mathrm{~kg} / \mathrm{m} 2$ (range18.2, 29.4). The median preoperative BMI was $20.92 \mathrm{~kg} / \mathrm{m} 2$ (range18.2, 29.4). Out of 25 patients $7(28 \%)$ had pancreatic neoplasms, 11 (44\%) had peri-ampullary neoplasms, 4 (16\%) had distal cholangio carcinoma and $3(12 \%)$ had chronic pancreatitis. Median follow-up of the group was 17 months (range, 6 - 42) (Table 1).

Six patients (24\%) who were pre diabetics and three patients who were new onset diabetics remained as diabetics and their hypoglycemic medication requirements remained static from the time of discharge till the time of assessment. Out of nineteen patients who were non diabetics preoperatively, three $(15.8 \%)$ progressed to develop diabetes and two developed IGT according to WHO criteria.

The non-diabetics (non-DM) were compared with new onset diabetics and IGT patients. There was no difference in demographic data between the two groups. Median

\begin{tabular}{|c|c|c|c|}
\hline & Non diabetics $(n=14)$ & $\begin{array}{l}\text { Post - operative diabetic and IGT } \\
\qquad(\mathrm{n}=5)\end{array}$ & $\mathbf{P}$ - Value \\
\hline Age (years) & $44(\mathrm{Q} 1=36, \mathrm{Q} 3=70)$ & $56(\mathrm{Q} 1=50, \mathrm{Q} 3=72)$ & $0.551^{*}$ \\
\hline Gender (M/F) & $6 / 8$ & $1 / 4$ & $0.263^{\star}$ \\
\hline Pre-operative BMI & $21.33(\mathrm{Q} 1=13.89, \mathrm{Q} 3=31.87)$ & $18.6(\mathrm{Q} 1=16.9, \mathrm{Q} 3=22.51)$ & $0.129 *$ \\
\hline Waist to hip ratio & $0.95(\mathrm{Q} 1=0.89, \mathrm{Q} 3=1.4)$ & $1.02(\mathrm{Q} 1=0.78, \mathrm{Q} 3=1.12)$ & $0.228 *$ \\
\hline Physical activity index & $2(\mathrm{Q} 1=2, \mathrm{Q} 3=3)$ & $2(\mathrm{Q} 1=1, \mathrm{Q} 3=2)$ & $0.172^{\circ}$ \\
\hline Pancreatic volume resected $(\mathrm{mean} / \mathrm{cm} 3)$ & $72(\mathrm{Q} 1=18, \mathrm{Q} 3=4-128)$ & $120(\mathrm{Q} 1=25, \mathrm{Q} 3=1045)$ & $1.000 *$ \\
\hline \begin{tabular}{|l} 
Indication for PD \\
$\cdot \quad$ Pancreatic carcinoma \\
$\cdot \quad$ Peri-ampullary and distal \\
cholangio carcinoma
\end{tabular} & $\begin{array}{c}4 \\
10\end{array}$ & $\begin{array}{l}2 \\
3\end{array}$ & $0.331^{\star}$ \\
\hline
\end{tabular}

Table 1. Predictors of immediate post-PD DM

•Chi square test

*Mann-Whitney U test

The Sri Lanka Journal of Surgery 2016; 34(4): 11-15 

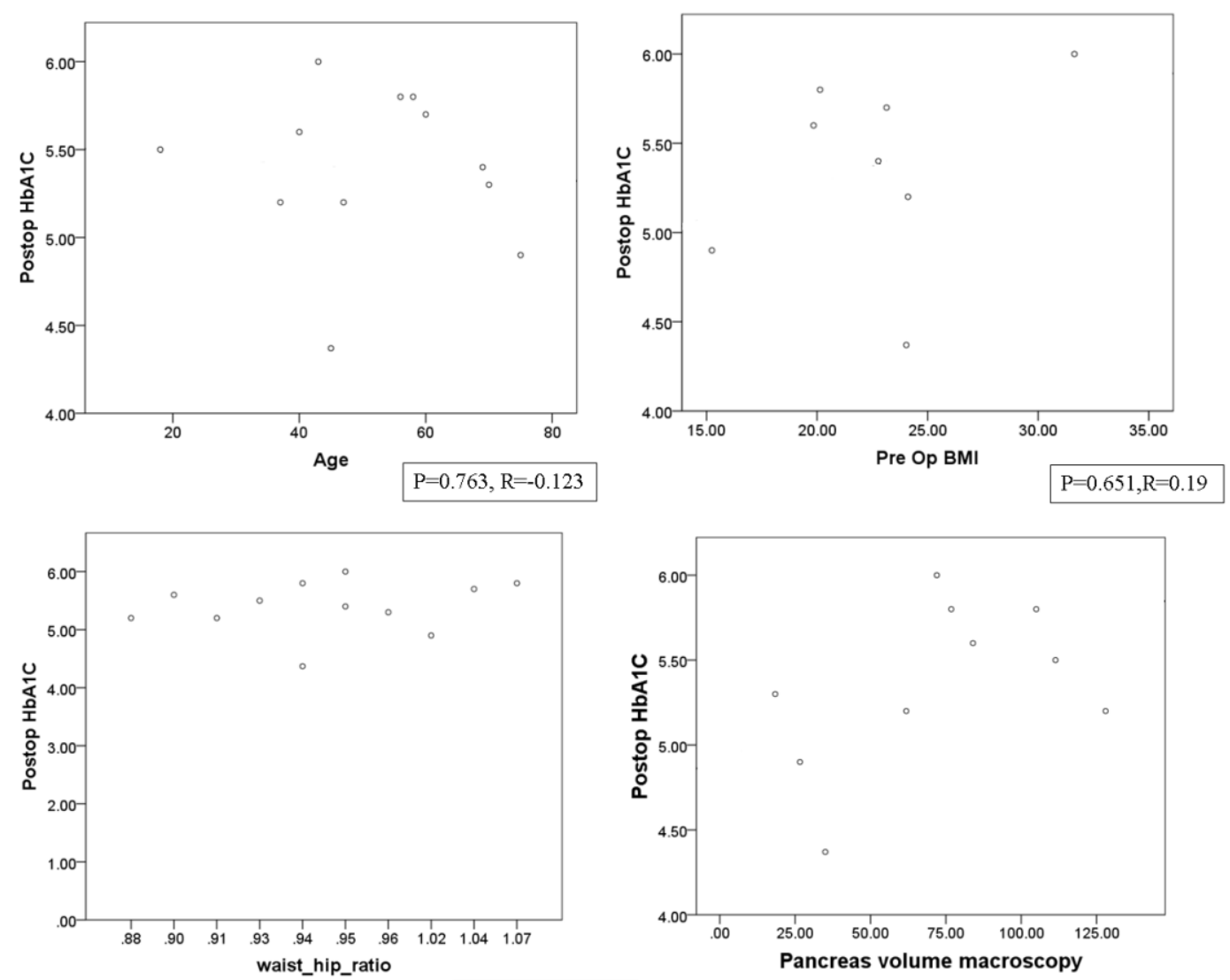

$\mathrm{P}=0.384, \mathrm{R}=0.276$

$\mathrm{P}=0.29, \mathrm{R}=0.3$

Figure 1. Correlation of $\mathrm{HbA1C}$ levels with a) Age b) preoperative BMI c) waist/hip ratio and d) pancreatic volume resected in immediate post-PD non diabetic group.
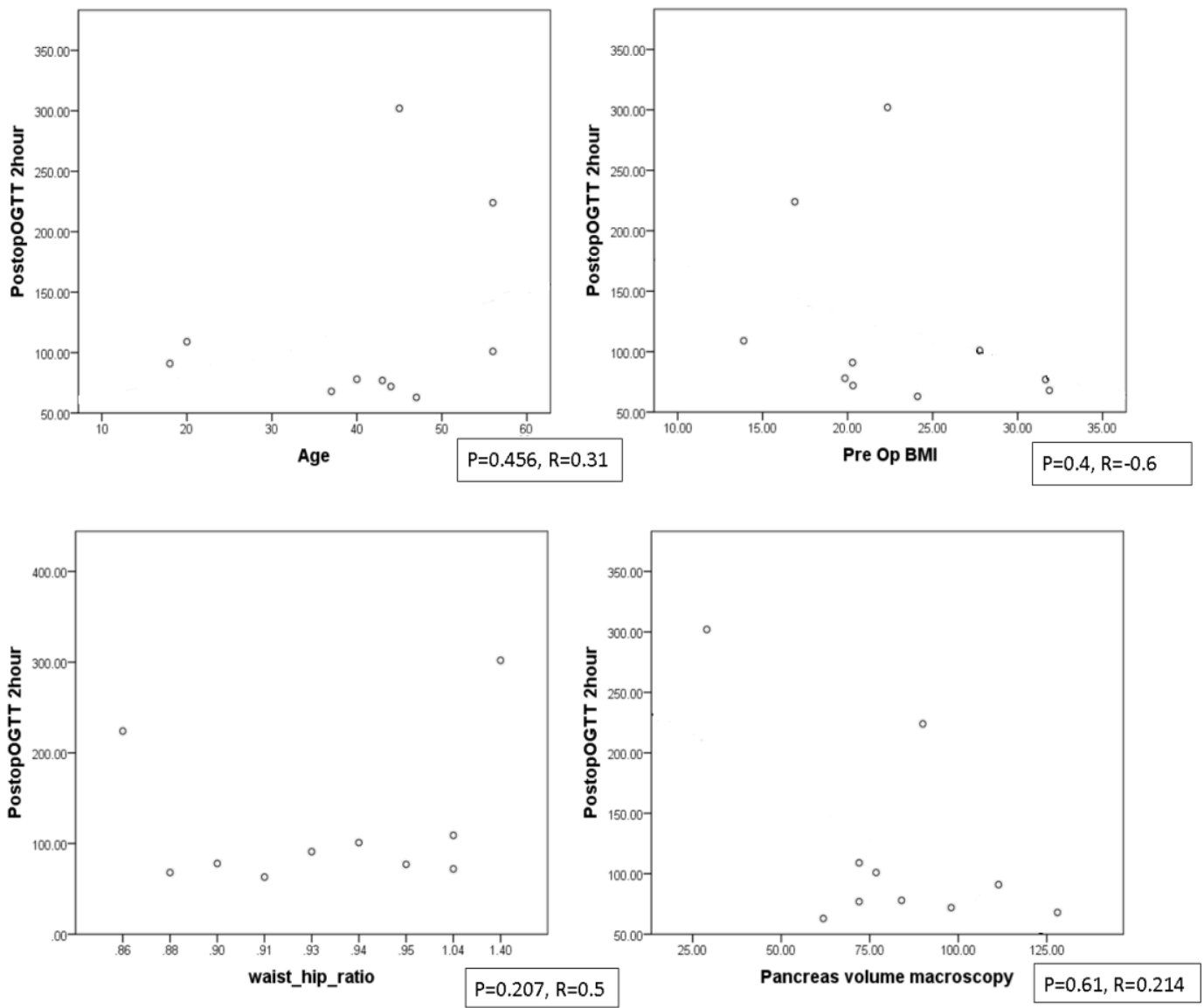

Figure 2. Correlation of OGTT levels at 2 hours with a) Age b) preoperative BMI c) waist/hip ratio and d) pancreatic volume resected in immediate post-PD non diabetic group. 
preoperative BMI was 22.9 and $19.8 \mathrm{~kg} / \mathrm{m} 2(\mathrm{P}=0.129)$ in post - PD non - DM and DM groups, respectively. There was no significant difference in body fat distribution, calculated median pancreatic volumes resected $(\mathrm{P}=1.000)$ or underlying pathology $(\mathrm{P}=0.331)$ for which $\mathrm{PD}$ was performed between the two groups (Table 1).

Eighty four percent (16/19, excluding IGT) patients remained as non-diabetics. In these patients $\mathrm{HbAlc}$ and two hour OGTT was compared with the potential risk factors to develop diabetes. There was no correlation of HbA1c level or two hour OGTT with age, preoperative BMI, waist to hip ratio and resected pancreatic volumes in the post-PD non-diabetic group (Figure 1 and 2).

Linear regression analysis was performed on OGTT and HbA1c levels separately using patient age, resected pancreatic volume, BMI, age and the waist to hip ratio as covariates. The models did not show a significant association with the factors (OGTT; $p$ value $=0.435$, HbA1c; $p$ value $=0.624$ ) and the individual variables did not show a significant association.

\section{Discussion}

After a median of 17 month follow-up, eleven patients who had diabetes ( 6 preoperatively and 5 new onset) had their hypoglycemic medication requirements static during the postoperative surveillance. Three patients had IGT. None of the factors evaluated predicted progression to diabetes or degree of glycaemic control.

Pancreatico-duodenectomy is known to affect glycaemic control in two ways. New onset diabetes is documented in up to $50 \%$ of the cases [6-8]. On the contrary, improvement of glycaemic control in a small percentage in previously known diabetics is also documented by others $[4,9,10]$. However, collectively, all of these studies have a common setback as they have evaluated PD and distal pancreatectomy together as a single group $[7,11,12]$. To the author's knowledge, at least two groups, have investigated the outcomes after the immediate perioperative period $[13,14]$. Nevertheless, we are of the opinion that the foregoing studies have intrinsic deficits.

First, assessing glycaemic control after PD and distal pancreatectomy as a single group is not rational due to the differences that occur in the normal physiology after PD due to unequal distribution of the beta cells in the pancreas. Second, immediate perioperative period is associated with many interacting confounders that can affect the glycaemic control.

A clear assessment of factors affecting glycaemic control can be accomplished only when patient's pancreas is provided an adequate period to return to normal homeostasis.
For instance, as shown here, there were 20\% new onset diabetics after PD. Hypoglycemic medication requirement remained static in new and previously known diabetics at 17 months. It must be noted however, that we have not monitored the glycaemic control in these patients with serial blood sugar or $\mathrm{HbA} 1 \mathrm{c}$ levels.

There are many risk factors that can potentially affect the development of diabetes after PD. It is important to detect potential risk factors pre operatively for patient preparation and long-term follow-up. In the current study those who developed post-surgical diabetes and the remaining counterparts did not show a major difference in possible risk factors that harbinger a future diabetic status. In previous data looking at long-term results after PD, You et.al [7] evaluated a sample of 55 patients with long term follow-up period of 60 months and assessed a number of risk factors for diabetes including the pancreatic volume.

They concluded that none of the factors including pancreatic volume predicted development of diabetes. While the studies of You et.al [7] concur with our findings it should be borne in mind that our diabetic sub-group was relatively small and a larger group of patients are required to arrive at definitive conclusions.

In another study by Hirata et.al (11) pre-operative HbA1c level was significantly higher in patients who developed immediate post-operative diabetes and yet again confirmatory data are needed to ascertain the validity of this finding.

In our cohort, $11 / 25(44 \%)$ were diabetics by the time they were discharged. After this initial peak there were no new onset diabetics at 17 months of follow-up. In the remaining group and non diabetics, there was no correlation with the OGTT values and the HbAlc levels with the evaluated potential risk factors. Even though our sample is relatively small as it is a highly selective sample this seems to be an interesting finding worthy of further exploration

As seen in our study, a significant proportion of patients develop diabetes in the immediate perioperative period after PD. The current study as well as other have clearly indicated the difficulty in predicting this potential group of patients with a diabetic risk is probably difficult due the many interacting factors. Once the initial risk group is taken off others seem to be stable as far as the glycaemic control is concerned.

Longer term follow up studies may however yield more definitive data. In one long term study by Ishikawa et.al [15] evaluated a similar selected small group of 51 patients, over a period of 7 years.

They concluded that patients with normal OGTT remained 
normoglycemic throughout, and those with impaired OGTT ran a higher risk of developing diabetes in the initial period after surgery [15]. However, after this initial period they did not progress to develop diabetes.

In conclusion, significant proportion of patients develop diabetes in the short and medium term after PD. Reliable prediction of this group pre-operatively is difficult due to many interacting, confounding factors. Clearly these patients need to be closely monitored and managed during the short and medium term postoperative period to ascertain their diabetic risk.

All authors disclose no conflict of interest. The study was conducted in accordance with the ethical standards of the relevant institutional or national ethics committee and the Helsinki Declaration of 1975, as revised in 2000 .

\section{References}

1. Grace PA, Pitt HA, Tompkins RK, DenBesten L, Longmire WP. Decreased morbidity and mortality after pancreatoduodenectomy. Am J Surg. 1986; 151: 141-9 https://doi.org/10.1016/0002-9610(86)90024-3

2. American Diabetes Association, Drouin P, Blickle JF, Charbonnel B, Eschwege E, Guillausseau PJ, et al. Diagnosis and classification of diabetes mellitus. Diabetes Care. 2013; 36 Suppl 1:67-74. https://doi.org/10.2337/dc13-S067

3. Bays HE, Chapman RH, Grandy S. The relationship of body mass index to diabetes mellitus, hypertension and dyslipidaemia: Comparison of data from two national surveys. Int J Clin Pract. 2007; 61: 737-47. https://doi.org/10.1111/j.1742-1241.2007.01336.x

4. Basso D, Valerio A, Seraglia R, Mazza S, Piva MG, Greco E, et al. Putative pancreatic cancer-associated diabetogenic factor: 2030 MW peptide. Pancreas. 2002; 24:8-14. https://doi.org/10.1097/00006676-200201000-00002

5. NHS. The general practice physical activity questionnaire (GPPAQ). 2006;

6. Hamilton L, Jeyarajah DR. Hemoglobin A1c can be helpful in predicting progression to diabetes after Whipple procedure. HPB. 2007; 9: 26-8.

https://doi.org/10.1080/13651820600917286
7. Slezak La, Andersen DK. Pancreatic resection: effects on glucose metabolism. World JSurg [Internet]. 2001; 25: 452-60. https://doi.org/10.1007/s002680020337

8. You D Do, Choi SH, Choi DW, Heo JS, Ho CY, Kim WS. Longterm effects of pancreaticoduodenectomy on glucose metabolism. ANZJ Surg. 2012; 82: 447-51. https://doi.org/10.1111/j.1445-2197.2012.06080.x

9. Aggarwal G, Ramachandran V, Javeed N, Arumugam T, Dutta S, Klee GG, et al. Adrenomedullin is up-regulated in patients with pancreatic cancer and causes insulin resistance in beta cells and mice. Gastroenterology. 2012; 143: 1510-7 e1.

10. He XY, Li JF, Yao WY, Yuan YZ. Resolution of New-Onset Diabetes After Radical Pancreatic Resection Predicts Long-term Survival in Patients with Pancreatic Ductal Cell Adenocarcinoma. Ann Surg Oncol. 2013; 20: 3809-16. https://doi.org/10.1245/s10434-013-3095-2

11. Hirata K, Nakata B, Amano R, Yamazoe S, Kimura K, Hirakawa K. Predictive Factors for Change of Diabetes Mellitus Status After Pancreatectomy in Preoperative Diabetic and Nondiabetic Patients. J Gastrointest Surg. 2014; 1597-603. https://doi.org/10.1007/s11605-014-2521-5

12. White M a, Agle SC, Fuhr HM, Mehaffey JH, Waibel BH, Zervos EE. Impact of pancreatic cancer and subsequent resection on glycemic control in diabetic and nondiabetic patients. Am Surg. 2011; 77: 1032-7

13. Ferrara MJ, Lohse C, Kudva YC, Farnell MB, Que FG, ReidLombardo KM, et al. Immediate post-resection diabetes mellitus after pancreaticoduodenectomy: Incidence and risk factors. Hpb. 2013; 15:170-4. https://doi.org/10.1111/j.1477-2574.2012.00520.x

14. Yoshikawa A, Kozawa J, Okita K, Yoneda S, Okauchi Y, Uno S, et al. Preoperative insulin secretion ability and pancreatic parenchymal thickness as useful parameters for predicting postoperative insulin secretion in patients undergoing pancreaticoduodenectomy. Endocr J. 2012; 59: 383-92. https://doi.org/10.1507/endocrj.EJ11-0322

15. Ishikawa O, Ohigashi H, Eguchi H, Yokoyama S, Yamada T, Takachi K, et al. Long-term follow-up of glucose tolerance function after pancreaticoduodenectomy: Comparison between pancreaticogastrostomy and pancreaticojejunostomy. Surgery. 2004; 136: 617-23.

https://doi.org/10.1016/j.surg.2004.01.006 